\title{
Vibrating Wire Sensor Signal Denoising and Analysis based on HHT
}

\author{
DENG Xue ${ }^{1, a}$, WANG Tao ${ }^{2, b}$ \\ ${ }^{1}$ Beijing Institute of Technology, Beijing 100081, China \\ ${ }^{2}$ Beijing Institute of Technology, Beijing 100081, China \\ addxx_2013@163.com, bwangtaobit@bit.edu.cn
}

\begin{abstract}
Keywords: Vibrating wire sensor, Dynamic testing technique , HHT, Filtering
\end{abstract}
Abstract. Vibrating wire sensor has advantages of strong anti-interference ability, high sensitivity, easy to long-distance transmission, etc. However, currently only used for static testing technique, in geotechnical engineering and other fields. In order to make the advantages into full play, expand its application field and make it widely used in the areas of port crane, requires further study of dynamic testing technique. Based on research and analysis of static testing technique, the exploratory research of dynamic testing technique is being in popular. This paper creatively puts forward a method for filtering environmental interference and noise and on this basis, constructs an algorithm for dynamic testing, namely the HHT spectrum filtering algorithm based on wavelet threshold pretreatment. The new algorithm provides a viable direction for the vibrating wire sensors used for dynamic testing.

\section{Introduction}

Vibrating wire sensor, which is widely used by engineering has advantages of high sensitivity, strong anti-interference ability, low cable requirements, simple structure, etc ${ }^{[1]}$. However, due to the vibration mechanism of vibrating wire sensor, a frequency sweep motivation costs too much time, so it takes some time to complete a measurement cycle. Therefore, the industry at present mainly uses vibrating wire sensors do static testing, which means after one time of sweep excitation and frequency capture, it needs to wait for the vibrating wire coil completely still, and then next excitation and capture $^{[2]}$, unable to acquire signals in any time, greatly weakening the application scope of vibrating wire sensors. When it needs quick measurement, vibrating wire sensors do not meet the requirements, can only rely on resistance strain gauges and other sensors. Under the environment of long-term monitoring, however, the resistance strain gauge does not apply. And for those structures, which have installed the vibrating wire sensors, the replacement of the sensor will cause human and property losses. Thus, research on the mechanism of the dynamic testing is of great significance.

In terms of dynamic testing mechanism, it mainly divides into two parts: frequency sweep excitation and frequency capture, the excitation part shall be realized by hardware circuit. This paper mainly studies the signal processing process of time-varying signal obtained by the excitation part. Not only need to know the overall signal frequency components, but also need to understand each signal frequency component contained in every moment.

Currently on, the main analysis tools of non-stationary signals such as vibration signals are short-time Fourier transform, wavelet transform, Wigner Ville time-frequency distribution, etc ${ }^{[3]}$. Short-time Fourier transform is actually windowed Fourier transform, usually assumes that the signal in the effective duration of window function is stable, but this condition can not usually satisfy or approximate meet. Thus its time and frequency resolution is very low, can not meet the analysis requirements on the precision. Wavelet transform is a signal analysis method, which has developed in the last ten years, mainly used for data compression and image edge detection. Its shortcoming is that the mother wavelet is difficult to determine. However, the choice of the mother wavelet is crucial, almost a decisive factor in the success of wavelet transform. Wigner Ville time-frequency distribution has high time and frequency resolution. In addition to the good time-frequency gathering, Wigner Ville also has other good properties, suitable for analyzing non-stationary signals. However, there exists a cross-term in the time-frequency plane, seriously affected the time-frequency analysis results. 
Although the cross-term can be decreased by the kernel function, the time-frequency resolution reduced $^{[4]}$.

Chinese American N. E Huang and others make an in-depth study of the concept of instantaneous frequency, create a new algorithm named Hilbert-Huang transform (HHT), creatively put forward the new concept of Intrinsic Mode Function(IMF) and a method decomposing any signal into IMF named Empirical Mode Decomposition(EMD). Also, they put forward the reasonable definition, physical meaning and calculation method of instantaneous frequency, preliminarily establish a new time-frequency analysis method, which regards the instantaneous frequency and intrinsic mode signal as the indication of signal change and basic time-domain signal ${ }^{[5]}$. This methodology fundamentally gets rid of the bondage of the Fourier transform theory, showing unique advantages in practice.

This paper analyses the vibrating wire sensor signal by Hilbert Huang Transform, obtaining the Hilbert spectrum, instantaneous frequency and other useful information of measured vibration structure. On this basis, to improve and perfect the algorithm, the HHT spectrum filtering algorithm based on wavelet threshold pretreatment is proposed for signal filtering. Through the experiment results, it is seen this algorithm is feasible, being a worth extending frequency capture algorithm.

Hilbert - Huang transform. HHT mainly consists of EMD and Hilbert transform two parts. General signal contains a variety of oscillation modes, directly using Hilbert transform to get the instantaneous frequency is meaningless. The EMD decomposes complex signal to a combination of several IMF, which transformed by Hilbert can get meaningful instantaneous frequency and amplitude, both as a function of time ${ }^{[6]}$.

The fundamental principles of EMD. EMD is to determine instantaneous equilibrium position using the average value of the time series envelope and then decompose the nonstationary signal to a group of steady $\operatorname{IMF}^{[7]}$. Process a vibration signal X(t) by EMD. The main steps are as follows:

1) Find the maximum and minimum value of the vibration signal $X(t)$ and connect them by three times spline interpolation to get maximum envelope $X_{\max }(t)$ and minimum envelope $X_{\min }(t)$.

2) Calculate the average value $m(t)$ of $X_{\max }(t)$ and $X_{\min }(t)$

$$
m(t)=\left[X_{\max }(t)+X_{\min }(t)\right] / 2
$$

3) $X(t)$ minus $m(t)$ is to obtain a new series $h(t)$

$$
h(t)=x(t)-m(t)
$$

For different vibration sequences, the $h(t)$ satisfying next two conditions is $\operatorname{IMF}^{[8]}$ :

1) For a sequence of data, the numbers of extreme points and zero-crossing points are equal or differ by one at the most.

2) At any point, the average of envelope line formed by maximum and envelope line formed by minimum is zero.

The first condition is similar to the definition of the narrowband in traditional stationary Gauss process. The second condition forces the signal to the local symmetry by the requirement that the average of extremum envelope is zero. It excludes the instantaneous frequency fluctuation caused by the asymmetric waveform ${ }^{[9]}$. Suppose that the new data series $h(t)$ meeting two conditions above for the first time is $\mathrm{IMF}_{1}$. Name it after $\mathrm{C}_{1}(\mathrm{t})$. Generally speaking, $\mathrm{C}_{1}(\mathrm{t})$ represents the high frequency part in a vibration sequence. Namely, it is a vibration modal of the vibration sensor. Separate $\mathrm{C}_{1}(\mathrm{t})$ from the vibration sequence

$$
x(t)-C_{1}(t)=r_{1}(t) .
$$

Because the remainder still contains long period components, retreat it as the new sequence repeating the above steps

$$
r_{1}(t)-C_{2}(t)=r_{2}(t) \ldots r_{n-1}(t)-C_{n}(t)=r_{n}(t) .
$$


Until the remainder $r_{n}$ becomes a Monotone function or a constant. After EMD, $n$ IMF components $\left(\mathrm{C}_{1}, \mathrm{C}_{2} \ldots \mathrm{C}_{\mathrm{n}}\right)$ and a trend term or a constant $r_{n}$ are separated from the vibration sequence. The complete vibration sequence can be got if the IMF components are added on the trend term

$$
x(t)=\sum_{i=1}^{n} C_{i}(t)+r_{n}(t) .
$$

Hilbert transform. For an arbitrary time series $\mathrm{X}(\mathrm{t})$, the Hilbert transform result $\mathrm{Y}(\mathrm{t})$ can always be got $^{[10]}$

$$
Y(t)=\frac{1}{\pi} P \int_{-\infty}^{+\infty} \frac{X(\tau)}{t-\tau} \mathrm{d} \tau
$$

$\mathrm{P}$ is the main Cauchy component in the equation. According to the transform, $\mathrm{X}(\mathrm{t})$ and $\mathrm{Y}(\mathrm{t})$ can form a complex signal $\mathrm{Z}(\mathrm{t})$

$$
Z(t)=X(t)+i Y(t)=a(t) e^{i \theta t} .
$$

In Eq. 7

$$
a(t)=\sqrt{X^{2}(t)+Y^{2}(t)}, \theta(t)=\tan ^{-1}\left[\frac{Y(t)}{X(t)}\right] .
$$

Define the instantaneous frequency as

$$
\omega(t)=\frac{\mathrm{d} \theta(t)}{\mathrm{d} t} .
$$

Because the instantaneous frequency is a derivative of the phase function, there is no need to define the local frequency with the whole wave. Therefore, it avoids the defects that the high frequency signal is amplified and the low frequency signal is compressed by traditional analytical method.

The HHT spectrum filtering algorithm based on wavelet threshold pretreatment. The vibrating wire sensor is mostly used in bridge engineering, geotechnics and other outdoor environment. These external conditions have great influence on the sensor, so that the data measured always have noise, which will bring some errors on the Hilbert transform. There is a need to filter on the basis of original algorithm to improve the measurement accuracy. This paper provides a new method called the HHT spectrum filtering algorithm based on wavelet threshold pretreatment, which combines the advantages of wavelet filtering and HHT spectrum filtering to improve the operation precision.

The wavelet threshold filter. Wavelet analysis method has been widely used in the field of signal filtering. One typical method is threshold filtering based on orthogonal wavelet transform.

Suppose that a noisy one-dimensional signal expression is shown as follows:

$$
s(k)=f(k)+e(k), k=0,1,2, \ldots, n-1 .
$$

In the equation, $f(k)$ is the original signal, $e(k)$ is a Gauss white noise signal of high frequency, so $s(k)$ is a noisy signal. In the actual project, $f(k)$ is usually a relatively stable low frequency signal. The frequency components of the signal and noise have not much overlap. So it can be filtered by wavelet threshold filter. The steps are as follows:

1) Apply the wavelet transform to decompose the noisy signal to obtain approximation and detail coefficients of each layer.

2) Gauss white noise signals of high frequency mostly are distributed in detail section of high frequency. So a reasonable threshold can be set to deal with the wavelet coefficients. The coefficient is set to zero which is less than the threshold and the larger one is reserved.

3) Finally, restructure wavelet coefficients to recovery the original signal. 
HHT spectrum filter. The time-varying amplitude and instantaneous frequency of one IMF component can be obtained by formula (1) to (9). Then a collection is obtained containing frequency, amplitude and time as $\{F, A, T\}$. Therefore, the collection of all the IMF components is $S=\sum_{j=1}^{N}\left\{F^{j}, A^{j}, T^{j}\right\}$. N is the number of IMF components. The signal is processed through discretization in actual operation and suppose the signal has $M$ sampling points, so $S=\sum_{j=1}^{N} \sum_{i=1}^{M}\left\{F_{i}^{j}, A_{i}^{j}, T_{i}^{j}\right\} . \mathrm{S}$ can be shown through the gray encoding diagram. It's just the Hilbert

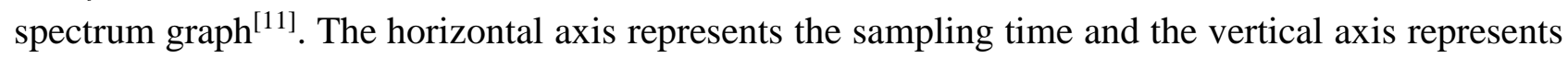
the instantaneous frequency. The shades of grey points in the figure represent the amplitude.

The two-dimensional grey graph of Hilbert spectrum is mapped from all the $\operatorname{IMF}_{\mathrm{j}}(1 \leqslant \mathrm{j} \leqslant \mathrm{N})$. Every grey point is originated from the value of one IMF at a certain time. There are $\mathrm{N}$ data points of Hilbert spectrum graph in the vertical direction on each time point and there are $\mathrm{N} \cdot \mathrm{M}$ data points in the whole two-dimensional grey graph. The gray value of these data points is corresponding to the amplitude. In fact, these data points are mapped from $S$ matrix straightly ${ }^{[12]}$. The IMF component, sampling time and amplitude originated from these data points can be got by the upper monitor. So a region in the two-dimensional grey graph can be drawn to trace back the earlier points in the IMF component, which forms a collection as $S_{1}$. Delete $S_{1}$ from $S$ (Set the corresponding points to zero): $S_{2}=S-S_{1}$. Then compose every IMF component in $S_{2}$ to achieve instantaneous frequency filtration of a given area in the Hilbert spectrum graph. It's called instantaneous frequency filtering algorithm based on Hilbert spectrum.

HHT spectrum filter based on wavelet threshold pretreatment. In the actual situation, a lot of noise interferences participating in decomposing will affect the result. The amount of IMF components will increase and the cumulative error caused by the end effect will also further increase, which is not favorable for filtering. So before being EMD decomposed, the signal can be treated by wavelet threshold pretreatment. It can improve the quality of EMD decomposing, reduce unnecessary IMF decomposition level, reduce the boundary accumulation effect of EMD decomposition, eliminate the modal aliasing phenomenon caused by local interference and improve the timeliness and accuracy of EMD effectively. Therefore, this paper puts forward a new filtering method called HHT spectrum filtering algorithm based on wavelet threshold pretreatment, which combines the advantages of wavelet filtering and HHT spectrum filtering. Firstly, the signal is preprocessed by wavelet threshold. Then, EMD decompose the signal after wavelet threshold pretreatment. Next, dispose the obtained IMF components by Hilbert transform and carry out HHT filtering on this basis. The process is shown in Fig. 1:

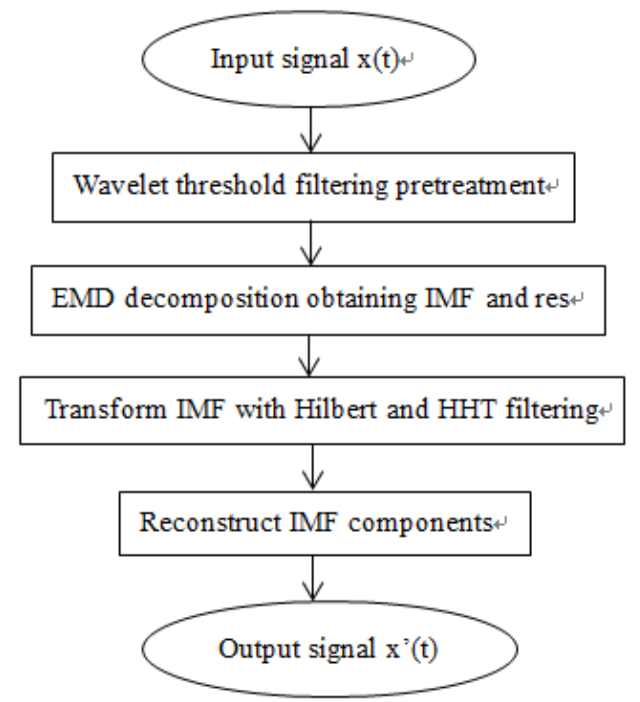

Fig.1: The process of HHT spectrum filtering algorithm based on wavelet threshold pretreatment 
Experiments and results. The experimental apparatus is shown in Fig. 2. Vibration table is JZ - 2 series made by the Beijing Wavespectrum Science and Technology Company, operating frequency range of $10 \sim 5000 \mathrm{~Hz}$. Vibrating wire sensor is BGK-4000 series produced by the United States Geokon corporation, sensitivity $1 \mu \varepsilon$.

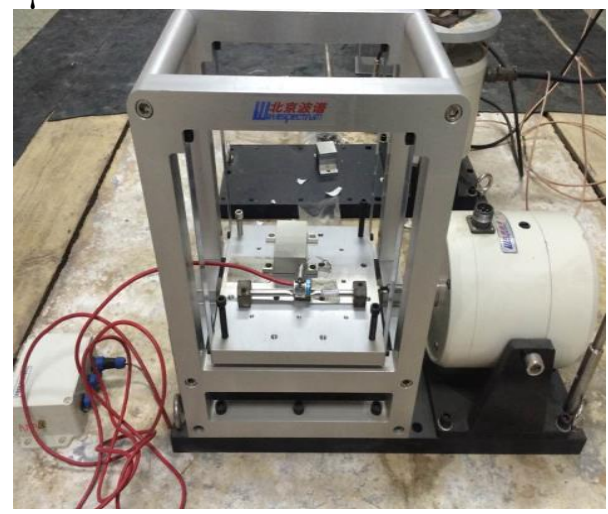

Fig. 2: The experimental apparatus

Put the vibrating wire sensor on the vibration table, fix the vibrating wire sensor. At this point, the vibration frequency of the sensor coil is equal to the vibration table. The vibration frequency of the vibrating table is set to $800 \mathrm{~Hz}$, means the vibration frequency of original signal $\mathrm{x}(\mathrm{t})$ is $800 \mathrm{~Hz}$. The output signal of the vibrating wire sensor is very weak, through amplified by hardware circuit, received by upper monitor software transmitted by the wireless network, the continuous dynamic sampling signal is as shown in Fig. 3.

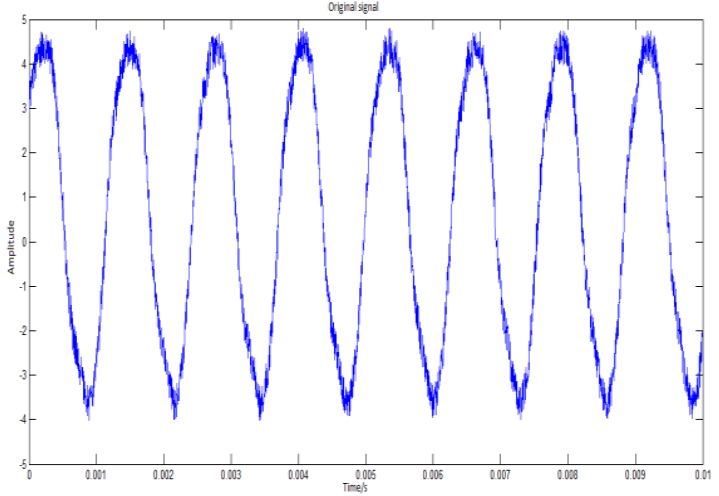

Fig. 3: original signal $x(t)$

Transform original signal $\mathrm{x}(\mathrm{t})$ with Hilbert to obtain Hilbert spectrum graph as shown in Fig. 4. In order to verify the advantages of HHT, comparing the results with the wavelet analysis, the time-frequency spectrum of original signal $x(t)$ after wavelet transform is shown in Fig. 5. By comparing Fig. 4 and Fig. 5, it is obvious to see that the wavelet analysis has poor time-frequency gathering, by contrast, HHT is better than the wavelet transform in time and frequency precision. But from the Hilbert spectrum graph, high-frequency noise existing in the original signal results in frequency calculation error, so the original signal need to denoise.

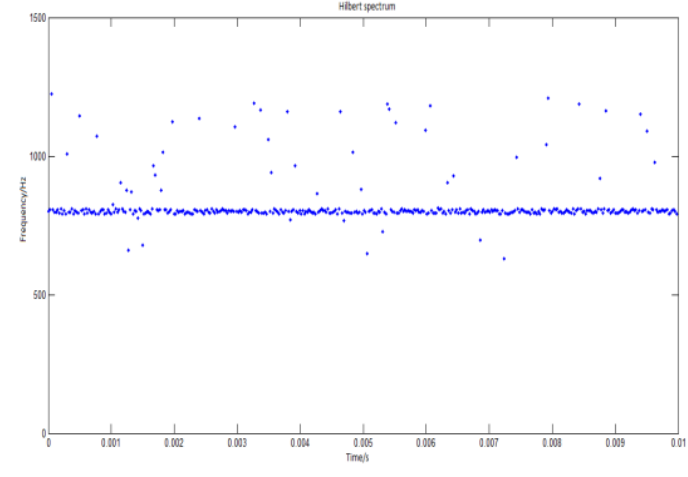

Fig. 4: Hilbert spectrum graph without filtering

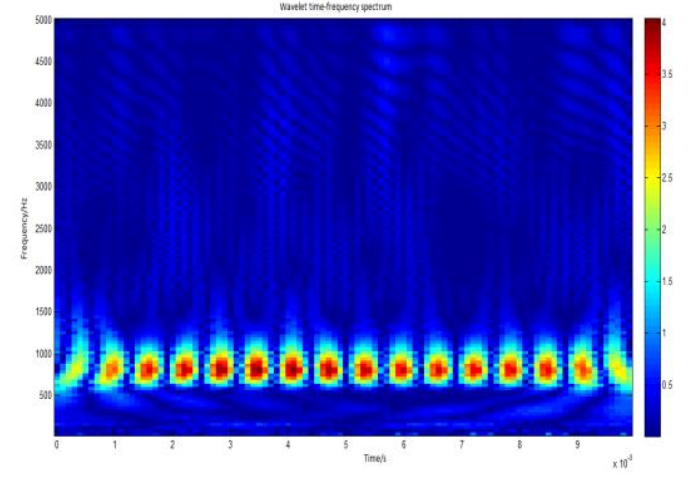

Fig.5: Time-frequency spectrum graph of wavelet transform 
Transform original signal $\mathrm{x}(\mathrm{t})$ with HHT spectrum filtering algorithm based on wavelet threshold pretreatment, Hilbert spectrum graph is shown in Fig. 6. By comparing Fig. 4 and Fig. 6, highfrequency noise decreases in the Hilbert spectrum graph after filtering.

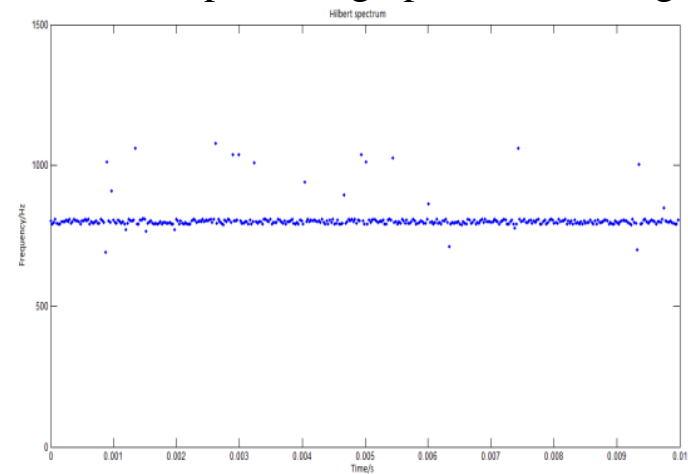

Fig. 6: Hilbert spectrum graph after filtering

Then observe the decomposition results of two types of EMD decomposition, shown respectively in Fig. 7 and Fig. 8 below. As can be seen from the figure, the first five components are highfrequency noise components, $\mathrm{IMF}_{6}$ is effective component, $\mathrm{IMF}_{7}$ is false component, res is residual component. Observe effective component $\mathrm{IMF}_{6}$, it is obvious to see that the latter result is more consistent with the original signal, decomposition effect is better.

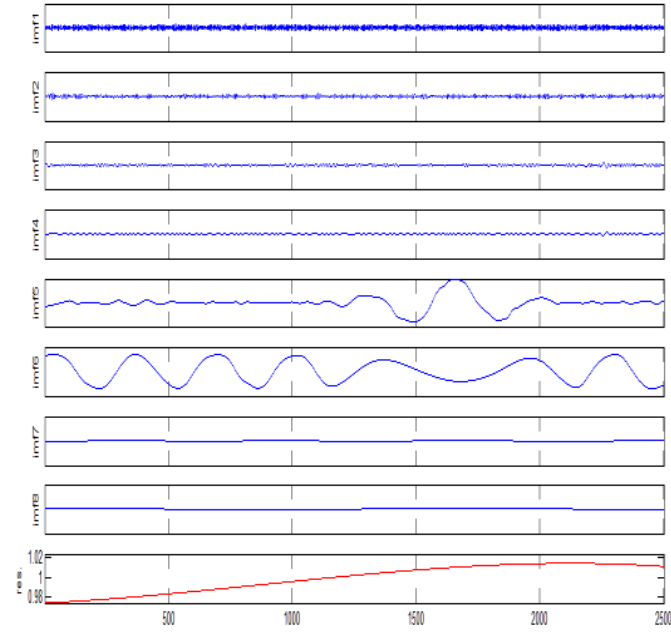

Fig.7: EMD decomposition results without filtering

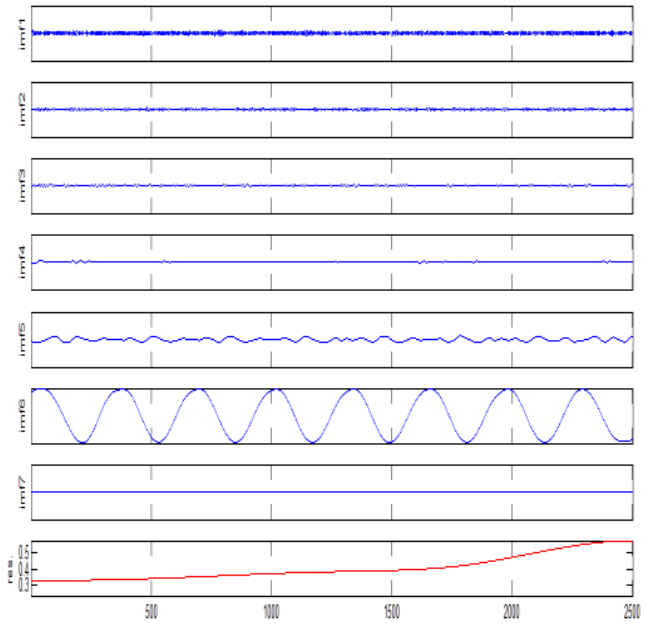

Fig.8: EMD decomposition results after filtering

Reconstruct IMF components after filtering, obtained a reconstructed signal as shown in Fig. 9, it can be seen that the high-frequency noise of reconstructed signal reduced a lot, the curve becomes smooth.

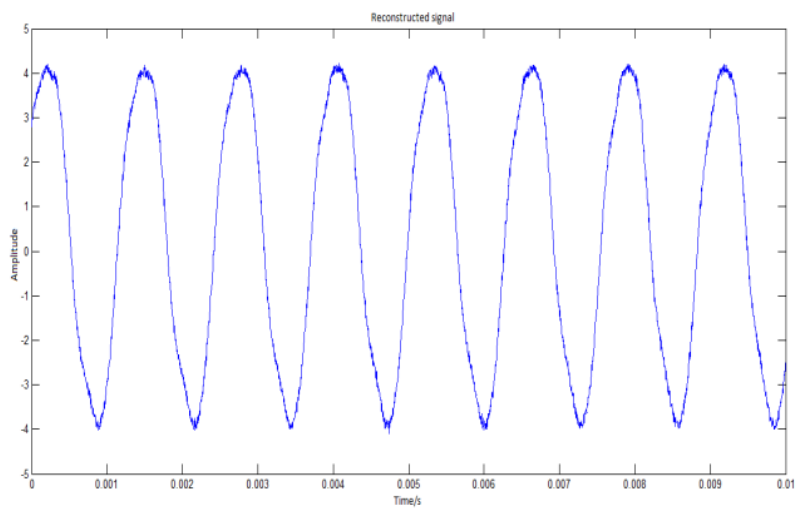

Fig. 9: Reconstructed signal 


\section{Conclusions}

In the background of vibrating wire sensor dynamic testing technique, the paper proposes a creative algorithm suitable for frequency capture of continuously varying non-stationary signals. Aimed at the noise signal occurring in real-world conditions, the HHT spectrum filtering algorithm based on wavelet threshold pretreatment is proposed. Experiments show that the algorithm is suitable for the frequency capture process of vibrating wire sensor with high accuracy, laid a solid foundation for the further development of dynamic testing technique. However, this algorithm also has disadvantages such as large computation, it needs to be improved in the future exploration.

\section{References}

[1] Santos. J and Janeiro. F. M: Development, implementation, and characterization of a standalone embedded viscosity measurement system based on the impedance spectroscopy of a vibrating wire sensor. Measurement Science and Technology. 26(2015), p.14

[2] Hsieh, Kai H, Halling, Marvin W, Barr and Paul J: Overview of vibrational structural health monitoring with representative case studies. Journal of Bridge Engineering, 11(2006), p.707-715

[3] Y. Li, H. L. Wei and S. A. Billings: Identification of Time-Varying Systems Using Multi-Wavelet Basis Functions. IEEE Transactions on Control Systems Technology, 19(2011), p. 656-663

[4] Leon Cohen in: Time-Frequency Analysis: Theory and applications, edtied by New York Prentice Hall, New York (1995), in press.

[5] Huang N. E, Shen Z and Long S. R: The Empirical Mode Decomposition and the Hilbert Spectrum for Nonlinear and Nonstationary Time Series Analysis. Proceedings of Royal society London A, (1998), p.903-995

[6] Wu Z. H and Huang N. E: Ensemble empirical mode decomposition: a noise assisted data analysis method. Advances in Adaptive Data Analysis, 1(2009), p.1-41

[7] Homborg. A. M and Van Westing. E. P. M: Novel time- frequency characterization of electrochemical noise data in corrosion studies using Hilbert spectra. Corrosion Science, 66(2013), p.97- 110

[8] CH Loh: Application of the empirical mode decomposition Hilbert spectrum method to identify near-fault ground- motion characteristics and structural responses. Bulletin of the Seismological Society of America, 91(2001), p.1339-1357

[9] Bandeira Soares. L and Bampi. S: A fast EMD-based technique for power quality signals decomposition, com- pression and time-frequency analysis. 2013 18th International Conference on Digital Signal Processing, Greece, July, 2013.

[10] Duggal. B. P and Kubrusly. C. S: A Putnam-Fuglede commu- tativity property for Hilbert space operators. Linear Algebra and Its Applications, 458(2014), p.108-115

[11] Shaojie Tang and Yi Yang: Interior tomography with radial Hilbert filtering and a priori information in a small circular area. Medical Imaging 2012: Physics of Medical Imaging, USA, 2012.

[12] Z. G. Zhang, Y. S. Hung and S. C. Chan: Local Polynomial Modeling of Time-Varying Autoregressive Models With Application to Time-Frequency Analysis of Event-Related EEG. IEEE Transactions on Biomedical Engineering, 58(2011), p. 557-566 